\begin{tabular}{lll}
\hline VOL. 81 & 1999 & NO. 2 \\
\hline
\end{tabular}

\title{
ON A GAP SERIES OF MARK KAC
}

BY

\section{KATUSI FUKUYAMA (KOBE)}

\begin{abstract}
Mark Kac gave an example of a function $f$ on the unit interval such that $f$ cannot be written as $f(t)=g(2 t)-g(t)$ with an integrable function $g$, but the limiting variance of $n^{-1 / 2} \sum_{k=0}^{n-1} f\left(2^{k} t\right)$ vanishes. It is proved that there is no measurable $g$ such that $f(t)=g(2 t)-g(t)$. It is also proved that there is a non-measurable $g$ which satisfies this equality.
\end{abstract}

1. Introduction. Let us recall the following result of Kac [3], which yields the central limit theorem for dyadic transformations.

THEOREM A. Let $f$ be a real-valued function with period 1 satisfying

$$
\int_{0}^{1} f(t) d t=0 \quad \text { and } \quad \int_{0}^{1} f^{2}(t) d t=1
$$

(1) If $f$ is of bounded variation or $\alpha$-Hölder continuous for some $\alpha>0$, then

$$
m\left\{t \in[0,1] \mid \frac{1}{\sqrt{n}} \sum_{k=0}^{n-1} f\left(2^{k} t\right) \leq x\right\} \rightarrow \Phi_{\sigma^{2}}(x),
$$

where $m$ denotes the Lebesgue measure and $\Phi_{\sigma^{2}}$ denotes the distribution function of the normal distribution with mean 0 and variance $\sigma^{2}$, i.e. $\Phi_{\sigma^{2}}(x)$ $=\int_{-\infty}^{x} e^{-u^{2} /\left(2 \sigma^{2}\right)} d u / \sqrt{2 \pi \sigma^{2}}$. Here, the limiting variance $\sigma^{2}$ is given by

$$
\sigma^{2}=1+2 \sum_{k=1}^{\infty} \int_{0}^{1} f(t) f\left(2^{k} t\right) d t<\infty .
$$

(2) If $f$ is of bounded variation or $\alpha$-Hölder continuous for some $\alpha>1 / 2$, then $\sigma^{2}=0$ if and only if $f$ is of the form

$$
f(t)=g(2 t)-g(t) \quad \text { a.e. }
$$

for some $g$ which has period 1 and is square integrable on $[0,1]$.

1991 Mathematics Subject Classification: 60F15, 42C15.

Key words and phrases: central limit theorem, gap theorem, cocycles. 
Earlier, Fortet [1] announced this result, but the proof was not complete. Kac succeeded in giving a rigorous proof, but he failed to prove part (2) for all $\alpha>0$. Instead of completing the proof, he gave the example below to show that part (2) does not hold without assuming any condition on $f$.

Example B. Put $c_{1}=1$ and $c_{j}=1 / \sqrt{j}-1 / \sqrt{j-1}$ for $j \geq 2$. Then the function $f(t)=\sum_{j=1}^{\infty} c_{j} \cos 2^{j} \pi t$ satisfies (1.2) with $\sigma^{2}=0$, but there is no integrable $g$ satisfying (1.4).

Having given the above example, Kac [3; p. 43] stated: "The question whether the representation (1.4) can be achieved in this case by means of a $g$ which is not integrable remains open".

In this paper, we give an answer to this question by showing the following theorem, which implies that there is no measurable $g$ satisfying (1.4) for the function of Example B.

THEOREM 1. Suppose that the Fourier coefficients $\widehat{f}(n)$ of $f$ are absolutely summable in $n$ and that $\widehat{f}(n)=0$ if $n \neq \pm 2^{k}(k=0,1, \ldots)$. If there is no square integrable $g$ satisfying (1.4), then there is no measurable $g$ satisfying (1.4).

On the other hand, for any given function $f$, it is always possible to construct $g$ satisfying (1.4), by using the Axiom of Choice. Of course this $g$ is not measurable in our case.

2. Proof of Theorem 1. First we prove a lemma and a proposition. Set $S_{n}(t)=\sum_{k=0}^{n-1} f\left(2^{k} t\right)$ and $\|h\|_{2}=\left(\int_{0}^{1}|h(t)|^{2} d t\right)^{1 / 2}$.

LEMma 1. Let $f$ be a square integrable function. Then there exists a square integrable $g$ satisfying (1.4) if and only if

$$
\liminf _{n \rightarrow \infty}\left\|S_{n}\right\|_{2}<\infty \text {. }
$$

P r o o f. If we assume (1.4), then (2.1) is trivial. We prove the converse. By (2.1) we can take a sequence $\left\{n_{j}\right\}$ of integers such that $\sup _{j \in \mathbb{N}}\left\|S_{n_{j}}\right\|_{2}<$ $\infty$. Let $g$ be the weak limit of $-S_{n_{j}}$ as $j \rightarrow \infty$. We see that $g(2 t)-g(t)$ is the weak limit of $f(t)-f\left(2^{n_{j}} t\right)$ as $j \rightarrow \infty$. By the Riemann-Lebesgue lemma, $f\left(2^{n_{j}} t\right)$ converges weakly to 0 as $j \rightarrow \infty$. Since the weak limit is unique, we have $f(t)=g(2 t)-g(t)$.

The following proposition plays the key role in the proof of the theorem.

Proposition 1. Assume the same conditions on $f$ as in Theorem 1 . If there is no square integrable $g$ satisfying (1.4), then

$$
\left\|S_{n}\right\|_{2} \rightarrow \infty \quad \text { and } m\left\{t \in[0,1] \mid S_{n}(t) /\left\|S_{n}\right\|_{2} \leq x\right\} \rightarrow \Phi_{1}(x) .
$$


P r o of. Since there is no square integrable $g$ satisfying (1.4), the first part of (2.2) follows from Lemma 1. The second part follows from the following theorem by Salem-Zygmund [4].

TheOREM C. Suppose that a sequence $\left\{\nu_{j}\right\}$ of positive integers satisfies the Hadamard gap condition:

$$
\nu_{j+1} / \nu_{j}>q>1 \quad \text { for all } j \in \mathbb{N},
$$

and that arrays $\left\{a_{n, j}\right\}_{j \leq j_{n}, n \in \mathbb{N}}$ and $\left\{b_{n, j}\right\}_{j \leq j_{n}, n \in \mathbb{N}}$ of real numbers satisfy

$$
A_{n}=\left(\frac{1}{2} \sum_{j=1}^{j_{n}}\left(a_{n, j}^{2}+b_{n, j}^{2}\right)\right)^{1 / 2} \rightarrow \infty \quad \text { and } \max _{j \leq j_{n}}\left(\left|a_{n, j}\right|,\left|b_{n, j}\right|\right)=o\left(A_{n}\right) \text {. }
$$

Then

$$
m\left\{t \in[0,1] \mid \frac{1}{A_{n}} \sum_{j=1}^{j_{n}}\left(a_{n, j} \cos 2 \pi \nu_{j} t+b_{n, j} \sin 2 \pi \nu_{j} t\right) \leq x\right\} \rightarrow \Phi_{1}(x) .
$$

Let $a_{n, j}$ and $-b_{n, j}$ be the real and imaginary parts of $2(\widehat{f}((j-n+1) \vee 0)$ $+\ldots+\widehat{f}(j))$ respectively. It is clear that

$$
S_{n}(t)=\sum_{j=0}^{\infty}\left(a_{n, j} \cos 2 \pi 2^{j} t+b_{n, j} \sin 2 \pi 2^{j} t\right)
$$

and $\left\|S_{n}\right\|_{2}=\left(\frac{1}{2} \sum_{j=0}^{\infty}\left(a_{n, j}^{2}+b_{n, j}^{2}\right)\right)^{1 / 2} \rightarrow \infty$. Clearly, $\left|a_{n, j}\right|$ and $\left|b_{n, j}\right|$ are bounded by $\sum|\widehat{f}(n)|<\infty$. Take $\left\{j_{n}\right\}$ satisfying $\sum_{j=0}^{j_{n}}\left(a_{n, j}^{2}+b_{n, j}^{2}\right) /\left\|S_{n}\right\|_{2}^{2}$ $\rightarrow 1$, and divide $S_{n}$ into two parts:

$$
S_{n}(t)=\left(\sum_{j \leq j_{n}}+\sum_{j>j_{n}}\right)\left(a_{n, j} \cos 2 \pi 2^{j} t+b_{n, j} \sin 2 \pi 2^{j} t\right) .
$$

If we normalize by dividing by $\left\|S_{n}\right\|_{2}$, thanks to Theorem C, the first part converges in law to the normal distribution. The second part converges to 0 in $L^{2}$-sense. Combining these, we have the conclusion.

Proof of Theorem 1. By Proposition 1, we have $\left\|S_{n}\right\|_{2} \rightarrow \infty$. Suppose that $f$ is represented by a measurable $g$ in the form (1.4). Then $S_{n}(t)=$ $g\left(2^{n} t\right)-g(t)$ and therefore, for $\varepsilon>0$, we have

$$
\begin{aligned}
m\left\{\left|S_{n}\right| /\left\|S_{n}\right\|_{2}>\varepsilon\right\} & \leq m\left\{\left|g\left(2^{n} t\right)\right|>\varepsilon\left\|S_{n}\right\|_{2} / 2\right\}+m\left\{|g(t)|>\varepsilon\left\|S_{n}\right\|_{2} / 2\right\} \\
& =2 m\left\{|g(t)|>\varepsilon\left\|S_{n}\right\|_{2} / 2\right\} \rightarrow 0,
\end{aligned}
$$

which contradicts the second formula of $(2.2)$.

3. Construction of $g$. Let us first introduce an equivalence relation $\sim$ on $[0,1)$ by $s \sim t$ if and only if there exist $n, m \geq 0$ such that $2^{n} s \equiv 2^{m} t$ $(\bmod 1)$. It is clear that each equivalence class $E$ satisfies $E \subset \mathbb{Q}$ or $E \subset \mathbb{Q}^{c}$. 
If we regard each element of $E$ as a vertex, and if we consider that we have an edge connecting $t$ and $s$ if $2 t \equiv s(\bmod 1)$, then $E$ has the structure of a graph. Since $t \notin \mathbb{Q}$ implies $2^{n} t \not \equiv t(\bmod 1)$, if $E \subset \mathbb{Q}^{c}$ then $E$ has no cycle and is a binary graph.

Now we are in a position to construct $g$. Take a representative $t_{0} \in E$ and put $g\left(t_{0}\right)$ arbitrary. Set

$$
g(t)= \begin{cases}g\left(t_{0}\right)+S_{n}\left(t_{0}\right) & \text { if } t=2^{n} t_{0}(\bmod 1), \\ g\left(t_{0}\right)-S_{n}(t) & \text { if } 2^{n} t=t_{0}(\bmod 1)\end{cases}
$$

where $n \in \mathbb{N}$. Since $E$ has no cycle, the function $g$ is well defined on $E$ and it satisfies $f(t)=g(2 t)-g(t)$ for any $t \in E$. Thus we can define $g$ such that $f(t)=g(2 t)-g(t)$ for any $t \in \mathbb{Q}^{\text {c }}$. If we define $g(t)=0$ for $t \in \mathbb{Q}$, we have $g$ satisfying (1.4).

\section{REFERENCES}

[1] R. Fortet, Sur une suite également répartie, Studia Math. 9 (1940), 54-69.

[2] K. Fukuyama, The central limit theorem for Riesz-Raikov sums, Probab. Theory Related Fields 100 (1994), 57-75.

[3] M. Kac, On the distribution of values of sums of the type $\sum f\left(2^{k} t\right)$, Ann. of Math. 47 (1946), 33-49.

[4] R. Salem and A. Zygmund, On lacunary trigonometric series II, Proc. Nat. Acad. Sci. U.S.A. 34 (1948), 54-62.

Department of Mathematics

Kobe University

Rokko, Kobe, 657-8501 Japan

E-mail: fukuyama@math.kobe-u.ac.jp 